\title{
Genetically modified organisms in food
}

UAB

Anna Pagès Aranda

Universitat Autònoma de Barcelona

21th June 2016

\section{Objectives}

- - - - - - - - - - - - - - - - - - - - - - - - - - -

(-Describe what GMOs are and the benefits and risks that can lead ?

I their presence in food.

- Research of legislation and control about GMOs

- Observe the current situation in the crops of GMOs

- Find out what consumers think, why and how it has been

influenced this opinion.

- Extract an opinion on GMOs based on data from reliable sources.

- Ex- - - - - - - - - - - - - - - - - - - - - - - - - - - - -

\section{Introduction}

\section{What are GMOs and how hey are obtained?}

What are GMOs and how they are obtained?

- an organism, with the exception of human beings, in which the genetic material has been altered in a way that does not occur naturally

- genetic modification occurs through the use of this techniques:

$\checkmark$ recombinant nucleic acid techniques

$\checkmark$ techniques involving the direct introduction into organism of heritable material

$\checkmark$ cell fusion (including protoplast fusion) or hybridization techniques

- - - - - - - - - - - - - - - - - - - - - -

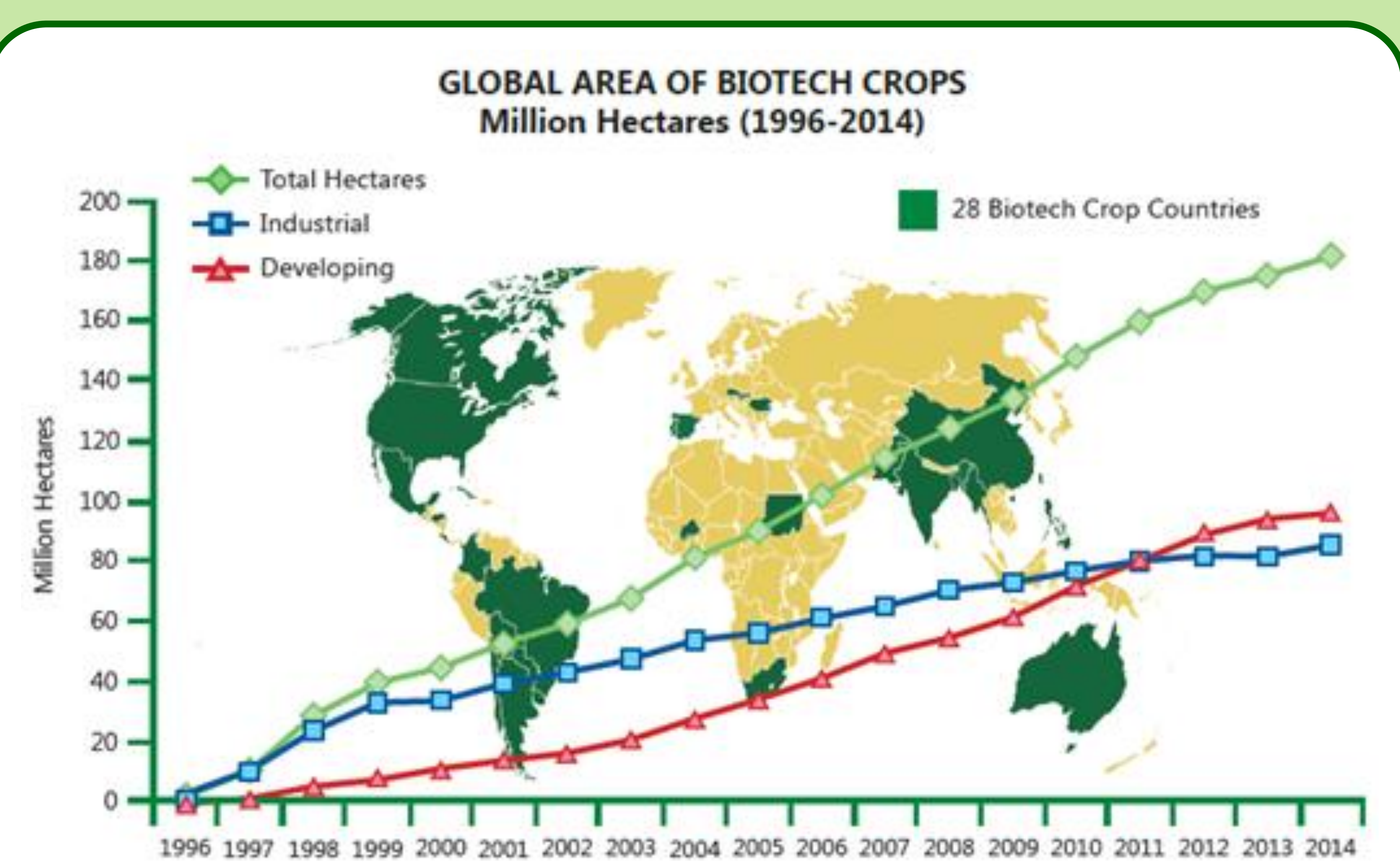

Figure 3. Evolution of the global area of biotech crops grown.

\section{Conclusions}

- The authorization process of GMOs in Europe is very demanding, where the products are exhaustively assessed.

- GMOs cultivation has increased over time, but in the recent years has stabilized.

- The introduction of GM foods in the food markets should be accompanied by appropriate policies to ensure consumer safety and better communicative tools in order to allow a reduction in the risk perceived by consumers.

- Must find ways to communicate the benefits and risks of GMOs to consumers without obstructions in order to let 1 them formulate there own opinions based on reliable data. 